\title{
La Evaluación de la Identidad Adoptiva: Traducción, Adaptación y Validación de la Entrevista para Adolescentes Adoptados (EAA-VE)
}

\author{
The Evaluation of Adopted Identity: Translation, Adaptation and Validation of the \\ Interview for Adopted Adolescents (EAA-VE)
}

\author{
María Mansilla Yuguero ${ }^{1}$, Marina Bueno Belloch² y María Teresa Coello García ${ }^{3}$
}

\begin{abstract}
Resumen
La proliferación de adopciones a nivel internacional ha generado una cantidad importante de investigación centrada en diferentes aspectos del proceso de adopción, principalmente para favorecer el bienestar psicológico de la persona adoptada. Entre las diferentes variables relacionadas con la salud psicológica del adulto adoptado, se encuentra la formación de la identidad adoptiva, la cual se manifiesta en las narraciones de adopción. En este trabajo, se presenta la traducción, adaptación y validación de una herramienta, Interview for Adopted Adolescents, que considera tanto las características de la narración como la posibilidad de contar con una medida cuantitativa. Para ello se ha entrevistado a 53 adolescentes de entre 11 y 19 años adoptados internacionalmente. Los resultados han puesto de manifiesto una adecuada confiabilidad interjueces de la versión española de la entrevista y el análisis de clústers ha distribuido a los participantes en 3 clústers que se han denominado identidad sin examinar, integrada y limitada-inestable.
\end{abstract}

Palabras clave: entrevista, adolescentes, identidad, adopción

\begin{abstract}
The increase of international adoptions has generated a significant amount of research focused on different aspects of the adoption, mainly to promote the psychological well-being of the adopted. Among the different variables related to the psychological health of the adopted adult, is the formation of the identity which can be seen in the adoption narratives. In this paper we present the translation, adaptation and validation of the Interview for Adopted Adolescents. This tool covers the narrative's characteristics and the possibility of relying on quantitative measurement. We interviewed 53 internationally adopted adolescents, aged 11-19 years. The results revealed an appropriate interjudge reliability of the Spanish version of the interview. Cluster analysis distributed participants in three clusters: identity without examination, integrated identity and limited-unstable identity.
\end{abstract}

Keywords: interview, adolescents, identity, adoption

\footnotetext{
${ }^{1}$ Psicóloga Clínica. Profesora Asociada. Facultad de Psicología. Universidad Complutense de Madrid. Campus de Somosaguas s/n, 28223. Madrid, España. Tel.: 913943123. Correo: mmansi01@ucm.es

2 Doctora en Psicología. Profesora Contratada Doctora. Facultad de Psicología. Universidad Complutense de Madrid. Campus de Somosaguas s/n, 28223. Madrid, España. Tel.: 913942832. Correo: marinabueno@psi.ucm.es

${ }^{3}$ Doctora en Psicología. Profesora Titular. Facultad de Psicología. Universidad Complutense de Madrid. Campus de Somosaguas s/n, 28223. Madrid, España. Tel.: 913942884. Correo: mtcoellogar@psi.ucm.es

Revista Iberoamericana de Diagnóstico y Evaluación - e Avaliação Psicológica. RIDEP · №57 · Vol.4 · 65-76 · 2020

ISSN: 1135-3848 print /2183-6051online
} 


\section{Introducción}

Si bien es cierto que, tras la I Guerra Mundial, empezaron las adopciones internacionales, no es hasta finales de la II Guerra Mundial (1939-1945) cuando aparece el fenómeno social de las adopciones internacionales dentro de un marco legal y político tal y como la conocemos en la actualidad. Estas adopciones estaban motivadas por una ayuda humanitaria y como respuesta de sensibilización con los problemas de la infancia más que por un deseo de tener hijos para aquellos que no pudieran procrear. Además de la segunda Guerra Mundial, otros conflictos o situaciones políticas, como la introducción por el gobierno chino de un solo niño por familia, provocaron también el impulso de la adopción internacional en general y de la adopción interracial en concreto.

Estas oleadas de adopción trajeron también consigo actuaciones abusivas, que llevaron a la redacción del Convenio de La Haya, relativo a la protección del niño y a la cooperación en materia de adopción internacional, hecho el 29 de mayo de 1993 y ratificado por España, entre otros países, el 2 de agosto de 1995. El fin de este convenio era adoptar medidas que garanticen que las adopciones internacionales tuvieran lugar en consideración al interés superior del niño y al respeto a sus derechos fundamentales, así como para prevenir la sustracción, la venta o el tráfico del niño (Martín, 2004).

Debido a esta mejora en las políticas de protección al menor, a la disminución de adopciones nacionales y al aumento de familias solicitantes de adopción, se producen en España entre 1997 y 2012 el mayor aumento en número de adopciones, siendo España el segundo país del mundo con más procesos de adopción, cerca de 65.000 , siendo la mayoría (75\%) en este periodo de carácter internacional (Fundación Foessa, 2014).

Este aumento de adopciones, a nivel internacional, redundó en un interés por parte de los investigadores del ámbito sanitario y social, en profundizar en diferentes aspectos vinculados al proceso de adopción. En concreto, las primeras investigaciones psicosociales se centraron en estudiar variables relacionadas con la llegada del niño al hogar y su adaptación inicial, tanto por parte de la familia como del niño (Berastegui 2007) y a comparar el ajuste psicológico entre adoptados y no adoptados en relación con las áreas del desarrollo físico, cognitivo y problemas de conducta (Juffer \& Van IJzendoorn, 2005; Beckett et al., 2003). Después, las investigaciones comenzaron a centrarse en las relaciones de apego (Grant-Marsney, Grotevant, \& Sayer, 2015; Román \& Palacios, 2011).

Posteriormente, con la llegada a la edad de la adolescencia de los niños adoptados, los investigadores centraron sus estudios en esta etapa del desarrollo, considerándola de especial interés, por los importantes cambios físicos, cognitivos y emocionales que se producen.

La adolescencia, en general, supone un periodo de mayor vulnerabilidad para el bienestar psicológico, principalmente por los acontecimientos vitales estresantes a los que tienen que hacer frente (Kushner, 2015). Estos acontecimientos, están presentes en mayor medida en los adolescentes adoptados, debido a las vivencias tempranas que pueden influir en el comportamiento y en el desarrollo de problemas psicológicos, sobre todo en problemas de tipo externalizantes como la hiperactividad, problemas de relación y problemas de conducta (Askeland, et al., 2017).

Estas dificultades a nivel psicológico pueden estar mediadas, tanto en el adolescente en general, como en adolescentes adoptados, por diferentes variables, como la identidad, la cual nos ocupa en el presente trabajo. Aunque la identidad no es en sí misma una medida de salud mental, sí que tiene implicaciones para las transiciones de desarrollo saludables durante la adolescencia y adultez emergente, así como para el bienestar psicosocial. De hecho, diferentes estudios centrados en la identidad adoptiva (Grotevant, Lo, Fiorenzo, \& Dunbar, 2017; Storsbergen, Juffer, Van Son, \& Hart, 2010) corroboran, mediante estudios longitudinales, que una no adecuada elaboración de la identidad adoptiva predice la presencia de problemas tanto internalizantes (e.g. síntomas depresivos) como externalizantes (e.g. problemas de conducta) en la vida adulta.

Además, cabe destacar que la relación entre la elaboración de diferentes dominios de la identidad y la salud mental está cobrando un auge importante en los últimos años. Así, se pueden encontrar estudios (Sevilla, Aparicio, \& Limiñana, 
2019) que refieren como las dificultades en la formación de la identidad, por ejemplo, en el dominio de género, pueden traer consecuencias de tipo psicológico.

La formación de la identidad ha sido conceptualizada, fundamentalmente, por el marco teórico formulado por Erikson (1963). Este autor define la identidad como un sentimiento de continuidad existencial en el tiempo y en el espacio, en el que, además, se da un reconocimiento de esa continuidad a través de otras personas. Así, la formación de la identidad sería un entrecruzamiento entre lo psicológico y lo social, entre lo individual y lo histórico (Erikson, 1971). Desde la inicial formulación de Erikson, la teoría de la identidad ha ido evolucionando hasta la actualidad, constituyendo la posibilidad del estudio empírico de dicho concepto una de las aportaciones más importantes que se han introducido en este campo. Dicha aportación fue formulada por Marcia a través de la entrevista semiestructurada Identity Status Interview (ISI) (Marcia 1966), en la que identificó la exploración y el compromiso como los dos procesos imprescindibles en la construcción de la identidad. Además, propuso una clasificación de cuatro estatus para caracterizar el desarrollo de un individuo hacia una identidad madura, denominados estados de identidad.

Posteriormente algunos autores sugirieron que el modelo de Marcia se alejaba del concepto de identidad personal de Erikson (Schwart, 2001) y, en consecuencia, aparecieron las corrientes neoeriksonianas que se acercaban más a la teoría de identidad formulada por Erikson e incluso iban más allá de los cuatro estatus de identidad que proponía Marcia. Estos modelos, basados en el enfoque constructivista, se han centrado principalmente en la exploración de la identidad, el compromiso y en cómo los estados de identidad resultantes se relacionan con el funcionamiento psicosocial. Dentro del enfoque constructivista se han desarrollado dos tipos de modelos. Por un lado, se encuentran los Modelos de Extensiones que complementan ampliamente la Teoría del Estatus de Marcia, estos modelos examinan facetas y añaden algún componente al marco de los estatus (Berzonsky, 1989; Grotevant, 1987 y Watterman, 1990), y por otro lado, se encuentran los Modelos de Expansiones, que van más allá de la formulación de Marcia, estos modelos consideran la identidad de forma más multidimensional que la sola intersección de la exploración y el compromiso (Adams \& Marshall 1996 ; Kurtines, 1999).

Desde los Modelos de Extensiones, se ha profundizado en el proceso de exploración y ha sido tratado como variable central para la formación de la identidad. Así, Grotevant (1987), propuso un modelo de proceso de exploración para conceptualizar la formación de identidad que consta de cuatro componentes: el primero se refiere a las características individuales, el segundo representa el proceso de formación de identidad dentro de un dominio específico (ocupación, ideas políticas, escuela, etc.), el tercero a los diferentes contextos de desarrollo (sociedad, la familia y los pares) en los que se construye el sentido de identidad del individuo y el último se refiere a las interdependencias que se desarrollan en los diferentes dominios de la identidad. Además, este autor señala que la exploración de la identidad puede ser conceptualizada en términos de cinco procesos que interactúan en el tiempo: 1) las expectativas y creencias del individuo; 2) la profundidad y la amplitud de la exploración; 3) la inversión afectiva realizada; 4 ) las fuerzas competidoras que pueden desalentar en la exploración y 5) las evaluaciones intermedias que determinarán si se realizarán nuevas exploraciones en la misma dirección.

Grotevant (1987) define la exploración de identidad como "una conducta de resolución de problemas dirigida a obtener información sobre uno mismo o sobre el entorno, para tomar una decisión sobre una elección de vida importante". (p.204)

Otras investigaciones han aportado conocimiento a este proceso de exploración de identidad. Así Kurtines (2001), basándose en la teoría constructivista, identificó tres procesos de resolución de problemas: la creatividad, la suspensión del juicio y la evaluación crítica, que facilitan el proceso de exploración durante la formación de la identidad. Meeus, Iedema y Maassen (2002), encontraron que, en el proceso de formación de la identidad, la exploración en profundidad y el compromiso están relacionados, es decir, aquellos adolescentes fuertemente comprometidos llevarán 
a cabo exploraciones más profundas en el dominio de elección. Otros autores (McLean, Syed, Yoder, $\&$ Greenhoot, 2014) también se han encontrado que, a través de la exploración de los roles y creencias de los diferentes dominios, es como el individuo llega a vivenciar un sentido de integración coherente y comprender su lugar en la sociedad.

Las investigaciones, además de centrarse en profundizar en el concepto de exploración, han estudiado los dominios de la identidad. Diversos autores proponen que la construcción de la identidad avanza a diferentes ritmos en diferentes dominios (Frisén \& Wängqvist, 2011; Grotevant, 1987 y Schwartz, 2001). Los dominios estarían interrelacionados, dándose una resolución secuencial de las crisis de identidad en todos ellos (Archer, 1989; Grotevant, 1987).

En referencia a los diferentes tipos de dominios cabe destacar que, aunque Erikson (1950) definió la ocupación e ideología como los dos dominios más importantes en los que la identidad se desarrolla, posteriormente diferentes autores agregaron nuevos dominios al concepto de identidad (McAdams, 1999; Marcia 1966) considerando el dominio ideológico dividido en los subdominios de identidad política y religiosa, y categorizándolos en dos grupos: los interpersonales y los ideológicos. La mayoría de los dominios investigados han sido aquellos sobre los que los individuos tienen algún control (ocupaciones, ideologías, valores, relaciones), sin embargo, los dominios sobre los que el individuo no tiene elección, como el sexo, la raza, la orientación sexual y el estado adoptivo, han sido menos estudiados (Grotevant, 1997).

Respecto a la exploración del dominio del estado adoptivo, tema que ocupa este trabajo, el individuo responde a las preguntas: "¿Quién soy yo como persona adoptada?", "¿Qué significa ser adoptado para mí?" y "¿Cómo encaja esto en mi comprensión de mi yo, familia y cultura?.

Este proceso de exploración de identidad como persona adoptada se denomina formación de la identidad adoptiva y generalmente va a suponer una construcción compleja porque su historia va a contener lagunas o realidades de difícil comprensión en momentos precoces del desarrollo (Ávila, 2005).
En la formación de la identidad adoptiva están implicados: un componente intrapsíquico, un componente que implica la relación intrafamiliar y un último componente que implica la relación con el entorno social más allá del ámbito familiar (Grotevant, 1997). La identidad adoptiva se manifiesta en las narraciones de adopción o historias que las personas adoptadas construyen sobre su sentido de persona adoptada, es decir, empiezan a reflexionar sobre el significado de ser adoptado e integrar sus pensamientos y experiencias en una narrativa coherente de identidad adoptiva (Grotevant, Dunbar, Kholer, \& Esau, 2000). A través del proceso de la narración, el sujeto crea identidad (McAdams, 2001), el sujeto tiene la oportunidad de dar sentido a sus propias experiencias y dar continuidad a su sentido de persona (McLean, 2008). Y es a partir de la última infancia y a través de la adolescencia cuando la narrativa de vida muestra una evidencia de narrativa de identidad bien formada (Habermas y de Silveira, 2008), siendo este momento evolutivo el comienzo formal del desarrollo de la identidad (Erikson, 1968, Habermas \& Bluck, 2000, McAdams, 2013).

Así, el estudio de estas narrativas se utiliza como herramienta de investigación para entender la función de la narración como una experiencia única e individual y para analizar la relación de estas narrativas con otras variables del individuo.

Por todo ello, se hace necesario contar con instrumentos que permitan explorar las características de la narración, pero que, además, ofrezcan una medida cuantitativa que permitan relacionar los estatus de la identidad con otras variables de funcionamiento psicosocial del individuo, ya que el trabajo de identidad adoptiva realizado por el adolescente va a determinar su bienestar psicológico (Brodzinsky, 1993). A pesar de la importancia de este tipo de medidas, en castellano no contamos con un procedimiento que permita medir estas características.

Una herramienta que considera tanto las características de la narración, como la posibilidad de contar con una medida cuantitativa, que además ha sido ampliamente utilizada en investigación sobre la identidad adoptiva (Grotevant, Dunbar, Kohler, \& Esau, 2000; Grotevant \& Von Korff, 2011; Von Korff \& Grotevant, 2011), es la Interview for Adopted 
Adolescents (IAA), (Dunbar \& Grotevant, 2004). Esta entrevista está basada en los fundamentos de la psicología narrativa (Grotevant \& Von Korff, 2011), pero permite codificar tres indicadores clave de la identidad adoptiva: a) exploración de identidad, que representa el proceso de desarrollo de la identidad; b) consistencia interna y c) flexibilidad, que representan coherencia narrativa (Von Korff, Grotevant, \& Friese, 2007).

El presente trabajo tiene como objetivo la traducción, adaptación y validación de la IAA (Dunbar \& Grotevant, 2004) en un grupo de adolescentes adoptados internacionalmente. Para llevar a cabo la validación de la entrevista se pretende calcular la validez de contenido y la fiabilidad interjueces.

\section{Método}

\section{Participantes}

Los participantes en este estudio fueron un total de 53 adolescentes adoptados internacionales residentes en España. Ellos y sus padres dieron su consentimiento informado para participar en el estudio. En el caso de los menores de edad el consentimiento fue facilitado por los padres y el asentimiento por los menores.

Los criterios de inclusión para participar en el estudio fueron los siguientes: la edad del adolescente adoptado oscilaba entre los 11 y 19 años, la adopción del adolescente tuvo lugar entre los 0 y 5 años de edad y la adopción fue internacional. Los criterios de exclusión que se plantearon fueron: que el adolescente presentase un diagnóstico neurológico, antecedentes de discapacidad intelectual $\mathrm{u}$ otras alteraciones del desarrollo e imposibilidad para hacer la entrevista presencialmente.

La recogida de datos se llevó a cabo entre los meses de febrero del 2015 y diciembre del 2016. Las familias adoptivas fueron contactadas a través de diferentes asociaciones de adopción ubicadas en todas las comunidades de España, que enviaron la información a las familias asociadas y aquellas que estuvieron interesadas contactaron con la persona responsable del estudio. En el primer contacto, 86 familias se interesaron en el estudio; de ellas: 10 familias $(11,6 \%)$ rechazaron participar por el temor de los padres a que la entrevista pudiera plantear al adolescente dudas y despertara sentimientos que hasta entonces no se hubieran dado, 16 familias $(18,6 \%)$ no participaron porque a pesar de que los padres si accedieron, el adolescente no quiso participar, 11 (12,7\%) familias no pudieron participar por no cumplir los criterios de inclusión.

Finalmente, en el estudio participaron 49 familias (56,9\%), y se entrevistó a 53 adolescentes, puesto que en 4 familias se entrevistó a 2 hermanos. De los adolescentes entrevistados la gran mayoría fueron mujeres (70\%), con edades comprendidas entre los 11 y 19 años, siendo la edad media de $14,15(\mathrm{SD}=2,36)$. La edad media de adopción fue de 3 años de edad. En relación al país de origen: el $47,2 \%$ de los adolescentes procedía de Asia, el $28,3 \%$ de África, el 18,9\% de Países del Este del Este y el 5,7 de países de América Latina.

\section{Instrumentos}

La Entrevista para Adolescentes Adoptados [Interview for Adopted Adolescents (IAA)] deDunbar y Grotevant, (2004) es una entrevista semiestructurada de 110 preguntas, diseñada para estudiar el desarrollo de la identidad en adolescentes adoptados, en los dominios de la elección de la ocupación (14 preguntas), las amistades (19 preguntas), las creencias religiosas o la espiritualidad (13 preguntas) y la adopción (64 preguntas). Respecto a la exploración de la identidad en el dominio de la adopción, la entrevista evalúa las siguientes categorías: a) profundidad (se refiere el grado en el cual el adolescente explora sobre el tema de la adopción, ésta se mide por el nivel de investigación sobre cualquier tema específico y tiene una amplitud de puntuaciones de 1 mínima a 4 profunda); b) afecto positivo y negativo (evalúa el nivel de afecto positivo o negativo en relación a la identidad adoptiva y tiene una amplitud de puntuaciones de 1 mínimo a 5 fuerte); c) relevancia (evalúa el nivel de relevancia que tiene la adopción para el adolescente, para ello se tiene en cuenta el grado de afecto vinculado a la identidad adoptiva y la relevancia de la identidad adoptiva en relación con el yo y otras identidades. La amplitud de puntuaciones de esta categoría va desde 1 mínima a 5 alta); d) consistencia interna de la narrativa (refleja la integridad del contenido de la narración y puede variar desde una teoría con lagunas y con 
poco fundamento, a una bien desarrollada e integrada. La amplitud de puntuaciones de esta categoría va desde 1 no existe teoría a 5 teoría bien documentada); y e) flexibilidad (implica el reconocimiento de que hay más de un punto de vista en la historia narrada y tiene una amplitud de puntuaciones de 1 baja a 5 alta).

\section{Procedimiento}

Para obtener la traducción y adaptación cultural de la IAA (Dunbar y Grotevant, 2004) se siguieron los pasos siguientes:

1) Se solicitó autorización al autor de la entrevista, Harold D. Grotevant, para utilizarla con adolescentes adoptados españoles.

2) La entrevista fue valorada por 3 expertos en adopción y evaluación psicológica que evaluaron en las 110 preguntas de la entrevista los siguientes aspectos; a) relevancia y representatividad de cada ítem, b) concisión y claridad de cada ítem y c) redacción. Cada uno de estos aspectos, para cada ítem, se puntuaron, para en una escala tipo Likert de 0 a 3 (deficiente, aceptable, bueno y excelente). Además, se realizó la traducción de la entrevista mediante la estrategia de traducción inversa (Hamblenton, 1996). Para ello, una persona bilingüe en ingles/español tradujo los ítems al español.

A partir de los datos obtenidos tras la valoración de los expertos, se calculó el grado de acuerdo entre estos, mediante el coeficiente kappa de Fleiss, véase Tabla 1, obteniendo grados de concordancia muy satisfactorios, ya que según Landis y Koch (1977) para 3 dominios sería casi perfecta. También, se obtuvieron resultados adecuados en la valoración de la relevancia y representatividad de cada ítem ( $\overline{\mathrm{X}}=2,5, \mathrm{SD}=0,3)$, concisión y claridad de cada ítem $(\overline{\mathrm{X}}=2,7 \mathrm{SD}=0,2)$ y redacción $(\bar{X}=2,2, S D=0,5)$, ya que se situaron entre valores buenos y excelentes. Estos valores no se han podido comparar con otros estudios previos puesto que no existen publicaciones sobre la entrevista que analicen estos aspectos.

Esto dio lugar a la versión 1 de la entrevista, que fue evaluada por 2 jueces independientes, bilingües en ingles/español para valorar la equivalencia semántica del instrumento. Esta versión, fue aplicada a 2 adolescentes adoptados e indicaron aquellas preguntas de la entrevista (4) que no comprendían fácilmente. Las cuáles se
Tabla 1. Grado de acuerdo interjueces para la totalidad de los ítems en la escala original

\begin{tabular}{lcc}
\hline Dominio & $\begin{array}{c}\text { Coeficiente } \\
\text { Kappa de } \\
\text { Fleiss }\end{array}$ & Concordancia \\
\hline Elección ocupación & .85 & Casi perfecta \\
Amistades & .97 & Casi perfecta \\
Creencias & .79 & Considerable \\
religiosas/espiritualidad & .97 & Casi perfecta \\
Adopción & .97 & \\
\hline
\end{tabular}

reformularon para facilitar su compresión (e.g. En la pregunta ¿Con quién sientes que puedes hablar de un modo más abierto y honesto acerca de la adopción? Se sustituyó la palabra honesto por sincero).

3) Finalmente, una persona bilingüe en inglés/español volvió a traducir la entrevista al inglés, comparando la versión original y la versión 1, dando lugar a la versión definitiva de la entrevista. De igual manera se llevó a cabo la traducción del Manual de codificación de identidad adoptiva (Grotevant, Dunbar, \& Kohler, 1999).

La versión definitiva de la entrevista, denominada Entrevista para Adolescentes Adoptados-Versión Español (EAA-VE), se aplicó a los adolescentes en una sola sesión con una duración media de 90 minutos. Cada una de las entrevistas fue registrada con una grabadora. En cada caso, antes de iniciar la entrevista, los padres cumplimentaban un cuestionario de datos diseñado ad hoc, que tenía como objetivo recoger información relativa a datos sociodemográficos tanto de los padres como del adolescente, así como de características relativas al proceso de adopción. Además, a los padres se les daba la oportunidad de revisar el protocolo de entrevista antes de entrevistar al adolescente. Una vez finalizadas las entrevistas, fueron transcritas textualmente por 2 graduados en psicología, entrenados previamente en el método de transcripción de Mergenthaler y Grill (1996).

Por último y para llevar a cabo la codificación de las entrevistas, se entrenó en la aplicación del Manual de codificación de identidad adoptiva (Von Korff, Grotevant, \& Friese, 2007) a 4 evaluadores independientes, psicólogos, y con experiencia previa en codificación de entrevistas. El entrenamiento se llevó a cabo durante 4 días, 3 horas cada día, en los que se revisaron los criterios de codificación y se realizaron 2 codificaciones 
piloto. Para valorar la concordancia entre los 2 evaluadores que codificaron cada entrevista, se obtuvo un valor del índice Kappa de .45 y .59, estos valores indicaron un grado de acuerdo moderado, lo que llevó a continuar con las codificaciones.

\section{Análisis estadístico}

Se realizó un análisis descriptivo para las variables demográficas y de caracterización sobre la adopción. Para evaluar la fiabilidad interjueces se utilizó el coeficiente Kappa de Cohen para cada categoría estudiada en el dominio de la adopción.

Para examinar la forma de agruparse los participantes en función de las puntuaciones obtenidas en las escalas se realizó un análisis jerárquico utilizando el método de vinculación media inter-grupo (ver el dendograma presentado en la Figura 1). En este método, en cada generación de clústers se calcula el promedio de la distancia euclídea al cuadrado entre todos los pares de elementos incluidos en los dos clústers comparados. Para examinar los perfiles de los clústers identificados y evaluar las potenciales diferencias entre los clústers en las escalas se utilizó el test de Kruskal-Wallis, utilizando la $d$ de Cohen como medida del tamaño del efecto para estas comparaciones. Se utilizaron los criterios de Cohen (Cohen, 1988) para la interpretación de la magnitud de los tamaños del efecto, representando una $d$ de Cohen de $.20, .50$ y .80 tamaños del efecto pequeños, medianos y grandes, respectivamente. Todos los análisis fueron llevados a cabo mediante el software SPSS versión 20.

\section{Resultados}

\section{Estadísticos descriptivos}

Para calcular la fiabilidad interjueces de la entrevista se utilizó el índice de Kappa ponderado, encontrando valores de .46 a .60 , siendo un grado de acuerdo moderado según la escala de valoración propuesta por Landis y Koch (1977).

La confiabilidad interjueces en las diferentes categorías, se calculó mediante el porcentaje de acuerdo (véase Tabla 2), obteniendo porcentajes de acuerdo entre el $83,3 \%$, en consistencia interna y el $100 \%$, en flexibilidad. Todos los porcentajes de acuerdo fueron superiores a los encontrados en la entrevista original (Dunbar y Grotevant, 2004), salvo el referido a consistencia interna, que es ligeramente menor en este estudio.

Tabla 2. Confiabilidad interjueces de la escala original y de la versión en español

\begin{tabular}{lcc}
\hline Categorías & IAA & EAA-VE \\
\hline Profundidad & $85.25 \%$ & $92.9 \%$ \\
Afecto positivo & $86.61 \%$ & $95.2 \%$ \\
Afecto negativo & $87.50 \%$ & $92.9 \%$ \\
Relevancia & $88.39 \%$ & $95.2 \%$ \\
Consistencia interna & $85.26 \%$ & $83.3 \%$ \\
Flexibilidad & $86.81 \%$ & $100 \%$ \\
\hline
\end{tabular}

\section{Análisis de clústers}

El dendograma (Figura 1) generado a partir del clúster jerárquico llevado a cabo sobre las puntuaciones obtenidas en las escalas dio como solución tres clústers: clúster $1\left(\mathrm{C}_{1}: n=20\right)$, clúster $2\left(\mathrm{C}_{2}: n=8\right)$, y clúster $3\left(\mathrm{C}_{3}: n=24\right)$.

De acuerdo a los resultados de las pruebas de Kruskal-Wallis presentados en la Tabla 3, los tres clústers presentaron diferencias significativas en todas las escalas: Profundidad $\left(\chi^{2}(2)=31,145\right.$, $p<.001)$, Afecto positivo $\left(\chi^{2}(2)=14,369, p=.001\right)$, Afecto negativo $\left(\chi^{2} \quad(2)=29,596, \quad p<.001\right)$, Relevancia $\left(\chi^{2}(2)=33,851, p<.001\right)$, Consistencia Interna $\left(\chi^{2}(2)=26,450, p<.001\right)$, y Flexibilidad en la narración $\left(\chi^{2}(2)=25,262, p<.001\right)$.

Un total de 20 participantes $(38,46 \%)$ se agruparon en el $\mathrm{C}_{1}$. Este clúster, que se ha denominado Identidad sin examinar, se caracteriza por bajas puntuaciones en todas las categorías. Los participantes de este grupo mostraron escasa exploración y relevancia en relación a la identidad de la adopción. Además, su narrativa se caracterizó por tener poca flexibilidad, una baja consistencia interna y bajo afecto. Así, los adolescentes no habían pensado en temas de adopción y cuando se les preguntaba por ello sus respuestas eran cortas y con escasa emoción. Cabe destacar puntuaciones muy bajas en Profundidad $(d=-3,33)$ y Consistencia Interna $(d=-3,03)$.

El $\mathrm{C}_{2}$ comprendió 8 participantes $(15,38 \%)$ y se caracterizó por puntuaciones elevadas en todas las facetas en comparación con los otros dos clústers, excepto en Afecto negativo, donde presenta puntuaciones más bajas que $\mathrm{C}_{3}(d=-.66)$. 


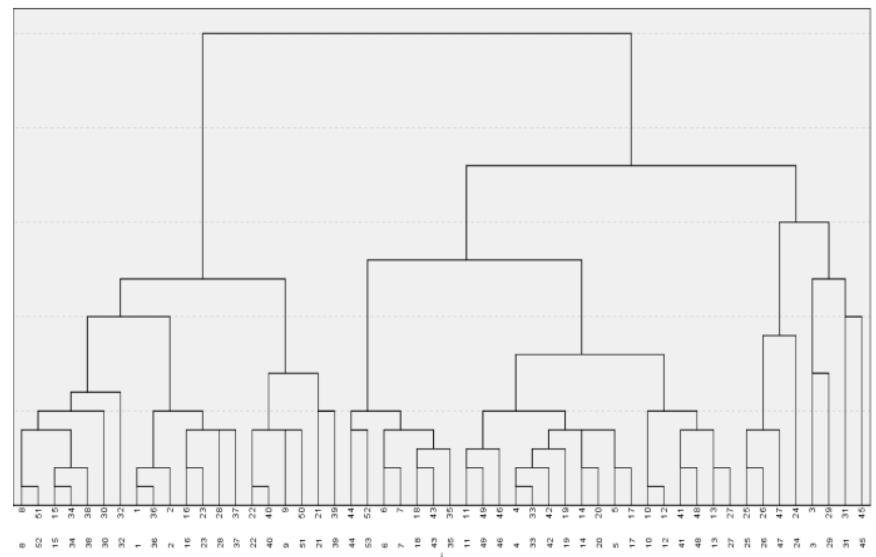

Figura 1. Dendograma correspondiente al clúster jerárquico sobre las puntuaciones obtenidas en las escalas de identidad adoptiva

Tabla 3. Perfil de medias y desviaciones típicas de los clústers en las escalas de identidad adoptiva y prueba de Kruskal-Wallis

\begin{tabular}{lccccccc}
\hline & $\mathrm{C}_{1}$ & $\mathrm{C}_{2}$ & $\mathrm{C}_{3}$ & & \multicolumn{2}{c}{$d$} \\
\cline { 2 - 8 } & $\mathrm{M}(\mathrm{SD})$ & $\mathrm{M}(\mathrm{SD})$ & $\mathrm{M}(\mathrm{SD})$ & $P^{*}$ & $\mathrm{C}_{1}$ vs $\mathrm{C}_{2}$ & $\mathrm{C}_{1}$ vs $\mathrm{C}_{3}$ & $\mathrm{C}_{2}$ vs $\mathrm{C}_{3}$ \\
\hline Profundidad & $1.70(.47)$ & $3.25(.46)$ & $3.00(.72)$ & $<.001$ & -3.33 & -2.14 & .41 \\
Afecto positivo & $2.70(1.17)$ & $4.38(.52)$ & $3.04(.75)$ & $<001$ & -1.85 & -.35 & 2.08 \\
Afecto negativo & $1.30(.47)$ & $2.38(1.19)$ & $3.04(.75)$ & $<.001$ & -1.19 & -2.78 & -.66 \\
Relevancia & $1.80(.41)$ & $3.88(.99)$ & $3.54(.83)$ & $<.001$ & -2.74 & -2.66 & .37 \\
Consistencia interna & $2.30(.57)$ & $4.25(.71)$ & $2.96(.55)$ & $<.001$ & -3.03 & -1.18 & 2.03 \\
Flexibilidad en la narración & $2.50(.76)$ & $4.13(0.64)$ & $3.00(.29)$ & $<.001$ & -2.32 & -.87 & 2.27 \\
$\mathrm{~N}$ & 20 & 8 & 24 & & & & \\
\hline
\end{tabular}

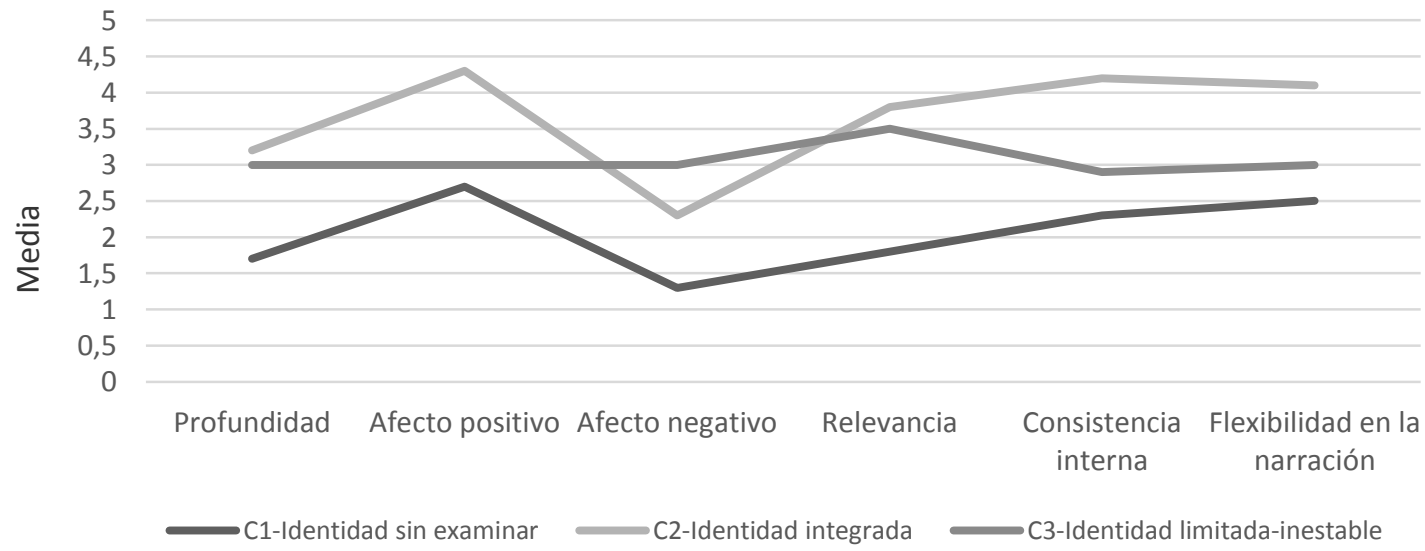

Figura 2. Perfil inter-clúster de identidad adoptiva

La narrativa de los participantes de este grupo mostró un afecto positivo y negativo moderado, además de una flexibilidad alta, reconociendo más de un punto de vista en la historia narrada. También este grupo manifestó una exploración de la identidad adoptiva de moderada a profunda. Así, los adolescentes de este grupo incorporaban aspectos negativos y positivos de su adopción y exploraban cómo la adopción se relacionaba con diferentes aspectos de su vida.

Un total de 24 participantes $(46,15 \%)$ se agruparon en el $\mathrm{C}_{3}$, denominado Identidad limitada-inestable. Este clúster se caracterizó por mayores puntuaciones en todas las facetas que el $\mathrm{C}_{1}$, especialmente en Profundidad $(d=-2,14)$, Afecto negativo $(d=-2,78)$ y Relevancia $(d=-2,66)$. Así los sujetos de este grupo mostraron una profundidad modesta y poca relevancia en la exploración de la identidad adoptiva, de tal forma que estaban abiertos a pensar sobre su adopción, pero no lo contemplaban como algo relevante en su vida. Además, la narrativa de estos 
adolescentes tenía escasa evidencia de apoyo. No obstante, los sujetos incluidos en el $\mathrm{C}_{3}$ presentaron puntuaciones menores que el $\mathrm{C}_{2}$ en todas las escalas salvo en afecto negativo, donde presentaron mayores niveles que $\mathrm{C}_{2}$. Así, los adolescentes de este grupo mostraron emociones negativas respecto a su adopción, en concreto exponían que se sentían incómodos hablando de su adopción con familiares y amigos, también cuando se exploraba la relación con su familia biológica. En la Figura 2 se presenta el perfil inter-clúster de identidad adoptiva.

\section{Discusión}

En el presente trabajo se ha traducido, adaptado y validado la versión española de la IAA (Dunbar \& Grotevant, 2004). Los resultados han puesto de manifiesto que la EAA-VE presenta unos porcentajes de confiabilidad interjueces adecuados en todas las categorías de la entrevista, siendo los porcentajes obtenidos superiores a la versión original de la entrevista salvo en la categoría de consistencia interna.

En la entrevista original, los autores (Dunbar \& Grotevant, 2004) describen cuatro clústers denominados; Identidad sin examinar (16,55\%), Identidad limitada (31,74\%), Identidad inestable (20,68\%) e Identidad integrada (31,03\%). Sin embargo, el análisis realizado en la EAA-VE, ha arrojado tres clústers denominados; Identidad sin examinar (38,46\%), Identidad integrada $(15,38 \%)$, Identidad limitada-inestable (46,15\%). Como se observa, en la EAA-VE la proporción de participantes que se agrupan en el clúster denominado Identidad limitada-inestable es la más elevada $(46,14 \%)$ y en la IAA, la mayor proporción correspondería a la Identidad limitada $(31,74 \%)$. Además, se encuentra una discrepancia entre la proporción de participantes que se distribuyen en el clúster Identidad sin examinar en la IAA $(16,55 \%)$ y en la EAA-VE $(38,46 \%)$. Es posible que esta situación esté relacionada con la edad de los participantes de ambos estudios, ya que los participantes de la IAA presentan una media de edad superior en un año a la edad media de los participantes del EAA-VE y como se ha visto en estudios anteriores (Dunbar \& Grotevant, 2004) los adolescentes más jóvenes con más frecuencia se agrupan en los clústers en los que la identidad adoptiva no está integrada.

La correspondencia en puntuaciones de las diferentes categorías (Profundidad, Afecto positivo, Afecto negativo, Relevancia, Consistencia interna y Flexibilidad en la narración) entre los clústers propuestos en la IAA y la EAA-VE es total en el clúster denominado Identidad sin examinar, ya que, en ambas entrevistas, es el clúster que presenta puntuaciones más bajas en todas las categorías.

En referencia al clúster Identidad integrada la correspondencia, entre ambas versiones de la entrevista, es igual para todas las categorías excepto para la de Relevancia, siendo en la IAA más baja que en la EAA-VE. En ambas versiones este clúster es el que arroja puntuaciones más altas en todas las categorías, excepto en Afecto negativo.

El clúster denominado en la EAA-VE, Identidad limitada-inestable, comparte características con los denominados en la IAA Identidad limitada e Identidad inestable. Así en las categorías de Profundidad, Afecto positivo, Relevancia, Consistencia interna y Flexibilidad en la narración, las puntuaciones obtenidas son similares a las del clúster de Identidad limitada de la escala original. Sin embargo, comparte con el clúster Identidad inestable de la escala original la puntuación más alta en la categoría afecto negativo.

Por todo ello, podemos concluir que la EAAVE es una herramienta con una adecuada confiabilidad interjueces y validez de contenido que nos permite evaluar el estatus de la identidad adoptiva en adolescentes a través de su narrativa. Contar con este instrumento de evaluación tiene diferentes implicaciones, en varios contextos, entre las que destacan; a) facilitar la investigación sobre la identidad adoptiva para poder seguir ampliando el conocimiento en este ámbito, b) posibilitar la evaluación, en el ámbito clínico, del estatus de la identidad adoptiva y c) desarrollar intervenciones psicológicas centradas en aquellos adolescentes que presentan estatus de identidad adoptiva que pueden favorecer la presencia de problemas psicológicos en la vida adulta. Además, estas implicaciones tienen un carácter muy amplio, ya que, a pesar de las particularidades de 
cada país, en materia de adopción, se puede utilizar en los diferentes países hispanohablantes.

No obstante, cabe destacar, que el presente trabajo muestra una limitación en relación con el tamaño de la muestra, puesto que no es todo lo amplio que se planificó en un primer momento ya que el acceso a la población es complejo. Aunque, es importante señalar que los estudios sobre exploración de identidad mediante entrevistas individualizadas presentan muestras similares e incluso menores a las presentadas en este estudio (Álvarez \& Jenaro, 2018). No obstante, convendría, en futuros trabajos, ampliarlo para dar más robustez a los datos obtenidos, así como ampliar el rango de edades.

\section{Referencias}

Adams, G. R., \& Marshall, S. K. (1996). A developmental social psychology of identity: Understanding the person-in-context. Journal of Adolescence, 19(5), 429-442. doi:10.1006/jado.1996.0041

Álvarez, P., \& Jenaro, C. (2018). Evaluación de la identidad del recluso mediante la técnica de la rejilla. Revista Iberoamericana de Diagnóstico y Evaluación - e Avaliação Psicológica, 47(2), 5-20. doi:10.21865/RIDEP47.2.01

Askeland, K. G., Hysing, M., La Greca, A. M. Aarø, L. E., Tell, G. S., \& Sivertsen, B. (2017). Mental health in internationally adopted adolescents: A meta-analysis. Journal of the American Academy for Child and Adolescent Psychiatry, 56, 203-213.

Archer, S. L. (1989). Gender differences in identity development: Issues of process, domain, and timing. Journal of Adolescence, 12, 117-138. doi:10.1016/0140-1971(89)90003-1

Ávila, A. (2005). La función parental en la adopción. Revista Iberoamericana de Diagnóstico y Evaluación - e Avaliação Psicológica, 1(19), 191-204.

Beckett, C., Castle, J., Groothues, C., O-Connor, T. G., Ruter, M., \& ERA Study Team (2003) Health problems in children adopted from Romania: Association with
Duration of deprivation and behavioural problems. Adoption and Fostering, 27(4), 1929.

Berástegui, A., \& Gómez, B. (2007). Ésta es tu historia: identidad y comunicación sobre los orígenes en adopción. Madrid: Universidad Pontificia Comillas.

Berzonsky, M. D. (1989). Identity style: Conceptualization and measurement. Journal of Adolescent Research, 4(3), 268-282. doi:10.1177/074355488943002

Brodzinsky, D. M. (1993). Long-term outcomes in adoption. The Future of Children, 3(1), 153166. doi: $10.2307 / 1602410$

Dunbar, N., \& Grotevant, H. D. (2004). Adoption narratives: The construction of adoptive identity during adolescence. In M. W. Pratt, \& B. H. Fiese (Eds.), Family stories and the life course: Across time and generations. (pp. 135161). Mahwah, NJ: Erlbaum

Erikson, E. H. (1963). Childhood and society. New York: Norton.

Erikson, E. H. (1968). Youth and crisis. New York: Norton.

Erikson, E. H. (1971). Identidad, juventud y crisis. Buenos Aires: Paidós.

Frisén, A., \& Wängqvist, M. (2011). Emerging adults in Sweden: Identity formation in the light of love, work, and family. Journal of Adolescent Research, 26(2), 200-221. doi:10.1177/0743558410376829

Fundación Foessa (2014). VII Informe sobre exclusion y desarrollo social en España. Caritas Española Editores: Madrid.

Grant-Marsney, H. A., Grotevanta, H. D., \& Sayer, A.G. (2015). Links between adolescents closeness to adoptive parents and attachment style in young adulthood. Family Relations, 64(2), 221-232. doi:10.1111/fare.1211

Grotevant, H. D. (1987). Toward a process model of identity formation. Journal of Adolescent Research, 2(3), 203-222. doi:10.1177/074355488723003

Grotevant, H. D. (1997) Coming to terms with adoption. Adoption Quarterly, 1(1), 3-27. doi:10.1300/J145v01n01_02

Grotevant, H. D., Dunbar, N., \& Kohler, J. K. (1999). Manual for coding identity in adopted adolescents. Unpublished manuscript. 
University of Minnesota, Minnesota -Texas Adoption Research Project.

Grotevant, H. D., Dunbar, N., Kohler, J. K., \& Esau, A. M. (2000). Adoptive identity: How contexts within and beyond the family shape developmental pathways. Family Relations, 49(4), 379-387. doi:10.1111/j.1741-3729.2000.00379.x

Grotevant, H. D., \& Von Korff, L. (2011). Adoptive identity. In S. Schwartz, K. Luyckx, \& V. L. Vignoles (Eds.), Handbook of Identity Theory and Research. (pp. 585-601). New York: Springer.

Grotevant, H.D., \& Lo, A.Y. (2017). Adoptive parenting. Current Opinion in Psychology, 15, 71-75. doi:10.1016/j.copsyc.2017.02.020

Habermas, T., \& Bluck, S. (2000). Getting a life: The emergence of the life story in adolescence. Psychological Bulletin, 126(5), 748-769. doi:10.1037/0033-2909.126.5.748

Habermas, T., \& de Silveira, C. (2008). The development of global coherence in life narratives across adolescence: Temporal, causal, and thematic aspects. Developmental Psychology, 44(3), 707-721. doi:10.1037/0012-1649.44.3.707

Hambleton, R.K. (1996). Adaptación de tests para su uso en diferentes idiomas y culturas: Fuentes de error, posibles soluciones y directrices prácticas. En J. Muñiz (Ed.), Psicometría (pp. 207-238). Madrid: Universitas.

Juffer, F., \& Van IJzendoorn, M. H. (2005). Behavior problems and mental health referrals of international adoptees: A meta-analysis. The Journal of the American Medical Association, 293, 2501-2515.

Kurtines, W. M. (2001). Human behavior and development: A coconstructivist perspective. Manuscrito no publicado.

Kurtines, W. M. (1999). A co-constructivist perspective on human behavior and development. Manuscrito no publicado, Florida International University, Miami

Kushner, S. C. (2015). A review of the direct and interactive effects of life stressors and dispositional traits on youth psychopathology. Child Psychiatry \& Human Development, 46(5), 810-819. doi:10.1007/s10578-014-0523-x
Landis, J. R., \& Koch, G. G. (1977). The measurement of observer agreement for categorical data. Biometrics, 33(1), 159. doi:10.2307/2529310

Marcia, J. E. (1966). Development and validation of ego-identity status. Journal of Personality and Social Psychology, 3(5), 551-558. doi:10.1037/h0023281

Martín, A. (2004). Legislación sobre acogimiento familiar y adopción. Normativa Internacional, estatal y autonómica. Madrid. Editorial Tecnos.

McAdams, D. P. (1999). Personal narratives and the life story. In L. Pervin \& O. John (Eds.), Handbook of personality: Theory and research (2nd Ed., pp. 478-500). New York: Guilford Press.

McAdams, D. P. (2001). The psychology of life stories. Review of General Psychology, 5(2), 100-122. doi:10.1037/1089-2680.5.2.100

McAdams, D. P. (2013). A psychological challenge for emerging adulthood, as illustrated in two notable case studies. Emerging Adulthood, 1(2), 151-158. doi:10.1177/2167696813481774

McLean, K. C. (2008). The emergence of narrative identity. Social and Personality Psychology Compass, 2(4), 1685-1702. doi:10.1111/j.1751-9004.2008.00124.x

McLean, K. C., Syed, M., Yoder, A., \& Greenhoot, A. (2014). The role of domain content in understanding identity development processes. Journal of Research on Adolescence, 26(1), 60-75. doi:10.1111/jora.12169

Meeus, W., Iedema, J., \& Maassen, G. H. (2002). Commitment and exploration as mechanisms of identity formation. Psychological Reports, 90(3), 771-785. doi:10.2466/PR0.90.3.771-785

Mergenthaler, E., \& Grill, S. (1996). Descripción de las reglas para la transcripción de sesiones de psicoterapia. Revista Argentina de Clínica Psicológica, 5(2), 163-176.

Roman, M., \& Palacios, J. (2011). Separación, pérdida y nuevas vinculaciones: El apego en la adopción. Acción Psicológica, 8, 99-111.

Sevilla, A. J., Evelio, M., \& Limaña-Gras, R. M. (2019). La salud de adolescentes y adultos transgénero: Revisión sistemática desde la 
perspectiva de género. Revista

Iberoamericana de Diagnóstico y Evaluación

- e Avaliação Psicológica, 1(50), 5-20.

doi:10.21865/RIDEP50.1.01

Schwartz, S.J. (2001). The evolution of Eriksonian and neo-Eriksonian identity theory and research: A review and integration. Identity, 1(1), 7-58.

doi:10.1207/s1532706xschwartz

Storsbergen, H. E., Juffer, F., van Son, M. J. M., \& Hart, H. (2010). Internationally adopted adults who did not suffer severe early deprivation: The role of appraisal of adoption. Children and Youth Services Review, 32(2), 191-197.

doi:10.1016/j.childyouth.2009.08.015.

Von Korff, L., \& Grotevant, H. D. (2011). Contact in adoption and adoptive identity formation: The mediating role of family conversation. Journal of Family Psychology, 25(3), 393-401.

doi:10.1037/a0023388

Von Korff, L., Grotevant, H. D., \& Friese, S. (2007). Manual for coding adoptive identity. University of Minnesota: Minnesota Texas Adoption Research Project, Department of Family Social Science. Manuscrito no publicado.

Waterman, A. S. (1990). Personal expressiveness: Philosophical and psychological foundations. Journal of Mind and Behavior, 11(1), 47-74. 